\title{
Peripheral injury alters schooling behavior in squid, Doryteuthis pealeii
}

Megumi Oshima, Marine Science Department, Coastal Carolina University, 301 Allied Drive, Conway, SC 29526

Theodor Di Pauli von Treuheim, Department of Bioengineering, Union College. 807 Union Street Schenectady, NY 12308

Julia Carroll, Biology Department, Williams College. 59 Lab Campus Drive, Williamstown, MA 01267

Roger T Hanlon, Program in Sensory Physiology and Behavior, Marine Biological Laboratory. $7 \mathrm{MBL}$ St Woods Hole, MA

Edgar T Walters, Department of Integrative Biology and Pharmacology, McGovern Medical School at UTHealth. 6431 Fannin St, Houston, TX, 77030

Robyn J Crook*, Department of Biology, San Francisco State University, 1600 Holloway Ave, San Francisco CA 94132

*Corresponding author. Email rcrook@sfsu.edu

Acknowledgements.

This work was supported by NSF grant number IOS-1146987 and IOS-1145478 to RJC, ETW and RTH, NSF grant number REU-1359230 to MO, TdP and JC, and startup funding from SFSU to RJC. 


\begin{abstract}
(200)
Animals with detectable injuries are at escalated threat of predation. The anti-predation tactic of schooling reduces individual predation risk overall, but it is not known how schooling behavior affects injured animals, or whether risks are reduced equally for injured animals versus other school members. In this laboratory study we examined the effects of minor fin injury on schooling decisions made by squid. Schooling behavior of groups of squid, in which one member was injured, was monitored over 24 hours. Injured squid were more likely to be members of a school shortly after injury (0.5-2 h), but there were no differences compared with sham-injured squid at longer time points (6$24 \mathrm{~h}$ ). Overall, the presence of an injured conspecific increased the probability that a school would form, irrespective of whether the injured squid was a member of the school. When groups containing one injured squid were exposed to a predator cue, injured squid were more likely to join the school, but their position depended on whether the threat was a proximate visual cue or olfactory cue. We found no evidence that injured squid oriented themselves to conceal their injury from salient threats. Overall we conclude that nociceptive sensitization after injury changes grouping behaviors in ways that are likely to be adaptive.
\end{abstract}

Keywords (3-6) Schooling, nociceptive sensitization, cephalopod, invertebrate, social behavior 


\section{Introduction}

Many animals aggregate to reduce their individual predation risk (Hoare et al., 2004; Thünken et al., 2014). In groups, risks are decreased due to increased scanning rates (Beauchamp, 2015; Roberts, 1996), predator confusion (Landeau and Terborgh, 1986; Olson et al., 2013) and decreased targeting risk for individuals (Creel et al., 2014). However, there are also costs to aggregation, including increased chances of detection by predators, increased parasite and disease transmission, and increased competition for food and mates (Herczeg et al., 2009). Thus, choosing to be a part of a group represents a dynamic risk/benefit tradeoff that each individual should make based on its own perceived vulnerability to predation versus other costs. Empirical studies of grouping behavior typically assume that individuals within groups have similar initial fitness; thus their cost/benefit tradeoffs are likely to be relatively homogenous. However, in any group of prey animals, initial individual fitness will vary based on previous life history, incorporating a range of conditions that may make individuals more or less vulnerable to predation relative to other group members.

The longfin inshore squid, Doryteuthis (Loligo) pealeii, is a mid-sized $(14-30 \mathrm{~cm}$ adult mantle length) pelagic species that, like many of the Loliginid species, often swims in groups. Groups may be either shoals (opportunistic aggregations) or schools (synchronized, directionally polarized groups), both of which occur across juvenile and adult life stages (Sauer et al., 1992).

Schools and shoals vary greatly in size, from fewer than 10 individuals to thousands, with large breeding aggregations of non-kin adults occurring annually during summer months off Cape Cod, Massachusetts (Shashar \& Hanlon, 2011). Observations of schooling dynamics in wild populations show that directional polarity and belt-shaped (i.e., linear) groups are common (Sugimoto and Ikeda, 2012). Composition of schools and individual position is highly fluid, with squid moving within, entering and leaving established schools readily, with seemingly little interaction between school members and 'enterers' or 'leavers' (Adamo and Weichelt, 1999). This suggests that school membership and position are primarily determined by the individual, and not by other members of the school or the school as a whole.

In captivity, 3-10 individuals readily congregate into small schools, with individuals typically matching each other's swimming speed and orientation. Captive squid also exhibit group resting in close proximity to each other, with similar cryptic coloration on sandy or rocky substrates. Squid are highly vulnerable to predation at all life history stages, typically from diurnally active fish predators (Staudinger and Juanes, 2010). Unusually for grouping species, squid are also under pressure from conspecific predation (cannibalism) (Adamo and Weichelt, 1999; Sauer and Smale, 1991) especially when a squid appears compromised. Thus the anti-predator advantages of schooling in squid are likely to be at least partially offset by significant costs, represented not only in terms of food and mate competition, increased detection by predators and increased parasitism risk, but also in risks from conspecific predation within a school or shoal.

We have demonstrated previously that in $D$. pealeii, minor injury produces a sensitized behavioral state characterized by visual and tactile hypervigilance (Crook et al., 2013, 2011). In many species (including squid), individuals who have experienced injury display a range of neural and physiological changes that produce long-lasting effects on behavior (Walters, 1994). After bodily injury in molluscs, plasticity in nociceptive sensory neurons produces a complex of sensitized behaviors that may include increased cutaneous sensitivity (Walters, 1987a), increased anxiety-like vigilance (Crook et al., 2011), decreased appetitive behavior (Walters et al., 1981) and enhanced defensive behavior (Crook et al., 2014). This sensitized behavioral state 
partially offsets the elevated risk of predation that occurs with sub-lethal injury (Crook et al., 2014). Despite extensive study of behavioral sensitization in both vertebrate and invertebrate species, few studies have addressed the functions of these changes in wild animals, and none have examined how nociceptive sensitization influences aggregation decisions in grouping species.

Here we examine how schooling behavior, a defensive tactic in some prey animals, is altered when one member of a small group has recently experienced a minor injury. Using the same species of squid (D. pealeii), which we have shown previously to gain a fitness advantage from nociceptive sensitization after minor peripheral injury (Crook et al., 2014), we ask whether an individual's decision to school with a group of uninjured conspecifics is changed by its minor injury. We also investigate whether injury affects schooling decisions when groups are presented with either a visual or olfactory predation threat.

\section{Methods}

\subsection{Animals}

Male and female adult squid (Doryteuthis [Loligo] pealeii), mantle length 9-22 $\mathrm{cm}$ ), were collected from local waters around Woods Hole, MA and maintained in groups of $10-15$ in holding tanks containing filtered ambient-temperature $\left(16-19^{\circ} \mathrm{C}\right)$, flow-through seawater. Fluorescent overhead lighting maintained a 12:12 day-night cycle. Squid were fed twice daily to satiation on small fish (Fundulus spp). Only animals with no evidence of previous injury were used. Squid were sexed by direct observation of the pink accessory nidamental gland through the ventral mantle (present in females and absent in males). Typically groups were composed of all females or females with one smaller male. Large males were excluded as they tended to disrupt schools with aggressive courtship behavior.

\subsection{Ethical note}

In the US invertebrate animals are not covered under IACUC regulations, thus no approval was required for this study. Manipulations of live squid followed the guidelines of the Animal Behavior Society for predator/prey studies (ABS, 2012) and the International Association for the Study of Pain for vertebrates undergoing potentially stressful and painful procedures. We chose to stage interactions and use a controlled injury procedure on selected captive squid because wild adult squid often have preexisting injuries of unknown age and varying severity, and observation of defensive interactions in wild marine animals is extremely difficult.

Animals were monitored daily and squid with any evidence of compromised health were euthanized immediately. All squid were euthanized at the conclusion of each trial by immersion in isotonic $\mathrm{MgCl}_{2}$ solution followed by decapitation and decerebration, and tissue was used in other physiological studies. To minimize animal numbers the study was designed only to detect large effects. Experimental injuries were minor compared to observed survivable injuries in the wild, the trial arena enabled effective squid camouflage (reducing escape swimming), and trials were kept short to reduce overall stress.

\subsection{Procedure}

\subsubsection{Arena}


The trial arena was a rectangular fiberglass tank, measuring $245 \times 120 \mathrm{~cm}$, filled with flow-through, filtered natural seawater to a depth of $45 \mathrm{~cm}$. The tank bottom was lined with a $3 \mathrm{~cm}$ deep bed of sand and small pebbles. Inflow and outflow pipes located on either side of the trial arena provided constant, predominately laminar flow through the tank with an exchange rate of approximately $40 \mathrm{~L} / \mathrm{min}$. Air pumped through an airstone provided constant aeration except during video recording.

\subsubsection{Focal animal treatment}

One squid in each group was selected as the focus of treatment and observation. It received one of two treatments 10 minutes prior to being placed into a schooling group of 4 or 5 other squid.

1. Control (C). Squid $(n=13)$ were restrained briefly in a net, placed onto a submerged surgical tray and then released. One fin $(L$ or $R)$ was arbitrarily designated as treated.

2. Injured (I): Squid ( $\mathrm{N}=29$ ) were restrained as above, and either the left or right fin received three closely spaced, $1 \mathrm{~s}$ duration crushes with serrated forceps. The injured area extended $\sim 10 \mathrm{~mm}$ rostral-caudally and $\sim 15 \mathrm{~mm}$ from the edge of the fin toward the body. This procedure approximates common natural injuries to the fins that occur during intraspecific aggression and hunting, and we have shown previously that this injury produces robust, long-lasting neural and behavioral sensitization (Crook et al., 2013; Crook et al., 2014)

All focal squid received a marking procedure, while other squid in the school were unmarked. A small volume $(0.1 \mathrm{ml}$ or less) of filtered (0.02 um pore diameter) seawater colored with Fast Green vital dye (Sigma-Aldrich) was injected subcutaneously into the distal midline tip of the mantle with a narrow (27 gauge) needle. Typically this injection produced no obvious behavioral response from the squid, and the dye remained visible for the $24 \mathrm{~h}$ observation period with minimal spreading.

\subsubsection{Threat cues.}

Trials were conducted under one of three conditions.

1. No external threats. Squid ( $n=13$ control, 14 injured) and their schooling groups ( 4 or 5 other unmanipulated squid per trial) were observed for 10 min periods at the following time points after injury: $30 \mathrm{~min}, 1 \mathrm{~h}, 2 \mathrm{~h}, 5 \mathrm{~h}, 6 \mathrm{~h}$ and $24 \mathrm{~h}$. Remote cameras recorded spontaneous behavior of the school.

2. Olfactory predator cue. Injured squid $(n=7)$ and their schooling groups (4 or 5 other unmanipulated squid per trial) were exposed to a transient olfactory predatory cue once during each observation period. A bucket (38 I) of seawater was collected from a shallow holding tank containing 10-15 individuals of two species of predatory fish, summer flounder (Paralichthys dentatus) and Black seabass (Centropristis striata). At the onset of each observation period, this conditioned seawater was added upstream of the trial tank and allowed to flow through the trial arena. Dye tests conducted with the same volume of water indicated that most of the added seawater flowed into the trial arena within one min, and most had flowed out of the tank by 2-3 min.

3. Visual predator cue. Injured squid $(n=8)$ and their schooling groups ( 4 or 5 other unmanipulated squid per trial) were exposed to a visual predator cue once during each 
observation period. A painted fiberglass model of a largemouth bass (Micropterus salmoides) was attached to a fishing rod. At the onset of the observation period the fish model was introduced to the arena by an experimenter out of view of the squid, and moved slowly across one side of the tank for $\sim 20 \mathrm{~s}$, then removed.

\subsubsection{Behavioral measures}

We defined a school as containing at least 3 squid, oriented in the same direction, and with all members spaced within one body length of each other (see Figure 1, top). The position of the focal squid was defined as either central (at least one squid between the focal squid and the school edge, irrespective of the number of squid in the school) or edge (Figure 1, bottom). When the focal squid was on the edge of the school, we recorded whether the treated fin faced outward away from the school, or inward toward the school.

\subsection{Data Analysis and Statistical Methods}

2.4.1. Data collection. Behavior of the schooling group was recorded with a single camcorder (Sony Handycam HDR-XR260). In each 10 min interval, a single point observation was recorded at the start of each minute. We recorded the following set of contingent variables at each point (see Figure 1 for descriptions of each variable):

1. Whether a school was present

2. If yes, whether the focal squid was part of the school

3. If yes, whether the focal squid was on the edge of the school,

4. If yes, whether the treated side was facing toward the school or away from the school

For each 10 min observation, we computed the proportion of "yes" observations, and then computed a grand mean of the proportions for each variable for "short term" (30min-2h) and "long term" (5h-24h) intervals after treatment of the focal squid.

\subsubsection{Statistical Analysis}

Proportionate data were arc-sine transformed and analyzed with non-parametric Kruskall-Wallis tests, followed by post-hoc Mann-Whitney-U tests. Comparisons were made between Control and Injured groups, and between injured groups with no predatory cues and Injured groups given either a visual or olfactory cue. Data are shown in figures as the mean of raw proportions +/- 1 SEM.

\section{Results.}

\subsection{Acute injury increases schooling probability in the absence of external threats}

3.1.1 Short term effects

In observations taken between 10 minutes and 2 hours after the introduction of an acutely-injured squid to a group of uninjured conspecifics, the presence of the injured squid significantly increased the proportion of time (Mann-Whitney- $U$ test, $p=0.002$ ) that a school was present, irrespective of whether the injured squid was a part of the school (Figure 2A). Among the groups where a threat cue was provided, probability of schooling was not different than in control groups, but was significantly less than for trials containing an injured squid without external threats (I vs. $\mathrm{O}, \mathrm{p}=0.048$, I vs V, $\mathrm{p}=0.007$, Figure 2A).

3.1.2 Long-term effects

In observations taken during the long-term phase (5-24h), there were no differences observed in schooling probability among any of the treatment groups (Figure 2B). 
3.2 Injury increases school membership for injured squid in the short term, and in the long term when in the presence of predatory threats.

3.2.1 Short-term effects

Shortly after the introduction of an injured squid to the school, injured squid were more likely to be members of schools compared with sham-treated squid ( $\mathrm{p}=0.01$, Figure $2 \mathrm{C}$ ), In the short term there were no differences in school membership among injured squid in the trials containing either no predatory cue, a visual cue or an olfactory cue (Figure 2C). 3.2.2 Long term effects

There was no difference in school membership between sham and injured squid at longer time points (Figure 2D). In the long term, injured squid were more likely to be members of a schooling group in the presence of either an olfactory or visual predator cue than in the absence of external threat cues (I vs $O, p=0.04$, I vs V, $p=0.03$, Figure 2D).

\subsection{Injured squids' position in a school is influenced by the modality of perceived} predatory threat.

3.3.1 Short term effects

In the short term, Injured squid were more likely to be located on the edge of a schooling group $(p=0.01)$ compared with control squids (Figure $3 \mathrm{~A})$, but there were no differences between the proportion of time injured squid spent on the edge of the school under the three threat conditions.

3.3.2 Long-term effects

In long-term observations, the higher probability that injured squid would be located on the edge of the schooling group persisted when compared with controls $(p=0.04$, Figure 3B). In contrast, at the long-term observation periods, there were significant differences in behavior of injured squids when exposed to either olfactory or visual threats, compared to injured squid with no threats present (Figure 3B). Injured squid exposed to an olfactory cue were more likely to be located on the outside of a schooling group compared with injured squid without cue exposure $(p=0.04)$, but injured squid exposed to a visual threat were significantly less likely to be located on the edge $(p=0.02)$.

3.4 Squid do not conceal injuries from salient threats.

We hypothesized that squid would attempt to conceal their injured fin from perceived threats - either other squid or an external predator. However, we observed no significant differences in injury orientation in any of the groups at either the short- or long-term time points (Fig 3C\&D).

\section{Discussion}

In this study we show that schooling decisions of $D$. pealeii are influenced by the presence or absence of injury within an individual and within a group. Further, there appear to be significant effects on schooling dynamics from interactions among injury, time since injury, and indicators of a predatory threat. In general, injured squid were more likely to join schools when their injuries were most likely to increase their risk of attack (i.e., in the immediate period after an injury was sustained, and over longer periods when predation risk was perceived to be high). Position within the school was affected both by the time since injury and by the type of threat cue presented, suggesting that complex and dynamic assessment of risk/benefit tradeoffs are made by injured animals. However, despite the clear ongoing influence of the injury on the squid's 
behavior, we found no evidence that squid were able to mitigate their predation risk further by concealing the site of their injury from perceived threats.

In this study we did not observe any instances of intraspecific predation, although cannibalism and attacks on smaller school members have been documented in wild squid (Adamo and Weichelt, 1999; Sauer and Smale, 1991) and in captive settings previously (Hanlon et al., 1983; Ibánez and Keyl, 2010). We fed squid individually to satiation twice daily and monitored groups for signs of aggression, stopping experiments when we observed low-level aggressive behaviors between group members. Likewise we did not measure predation risk directly by allowing groups of squid to interact with a live predator, thus the fitness benefits and costs of the schooling decisions made by injured squid were not quantified (although general reactions of this squid species to predators have been tested by Staudinger et al., 2011, and Crook et al, 2014). Nevertheless, we show that sublethal injury to only one member of a group has substantial effects on schooling dynamics, affecting not only the injured member but also changing the behavior of the group as a whole.

Similar to findings from studies of aquatic vertebrates, it appears that uninjured animals change their behavior in the presence of an injured animal (Krause, 1993; Lutterschmidt et al., 1994). An injured conspecific increased the likelihood that the squids would form a school, even if the injured animal was not a member of the schooling group. Uninjured squid may utilize olfactory or visual cues emitted by the injured conspecific to adjust their own behavior, similar to the well-documented effects of "shreckstoff," a chemical cue exuded from damaged skin of fish (Alves et al., 2013; Chivers et al., 2007). In molluscs, specialized defensive secretions have been implicated as alarm signals to conspecifics (Kicklighter et al., 2007; Walters and Erickson, 1986; Wood et al., 2008), but to our knowledge, the present observations provide the first evidence in this phylum that injured animals release chemicals that can function as cues. In previous studies using the same species, we showed that injured squid are preferentially targeted by fish predators (Crook et al., 2014), supporting our observations here that some type of cue is produced by injured squid. Given the small size of the injury relative to the squid's body and the absence of a visually conspicuous wound, it seems unlikely that the injured tissue itself is visible enough to be detected at a distance by a fish predator. In the present study, we found no evidence that squid attempted to conceal their wounded fin from either conspecifics or potential predators. If olfactory cues or visual "whole-body" cues are more important to predators than visual detection of wounds, attempting to conceal an injury from predators may provide little benefit.

Alternatively, evidence from physiological experiments (Crook et al., 2013) suggests orientation of a wounded area away from threats might be impossible for squid. In other molluscs (and mammals), nociceptive sensitization is strongest close to a wound, functioning in part to inform the nervous system of an injury's location on the body surface (Nikitin and Kozyrev, 1999; Walters, 1987b). In squid there is little evidence for site-specific sensitization; instead, the nervous system of the squid undergoes strong generalized sensitization (Crook et al., 2013) that is reflected in increased mechanical sensitivity over the entire body surface (Crook et al., 2011). We hypothesize that this form of plasticity may function to drive the generalized hypervigilance squid display after injury (which is strongly adaptive, see Crook et al., 2014), but it is unlikely to provide squid with a neural representation of an injury location on the body surface. Thus it is possible that squid do not orient their injury away from predators because their nervous systems are unable to integrate information on the location of the injury with the animals' body position; i.e., because they know only that they have received a significant injury, and not where the injury is. 
Although squid seem to lack the ability to conceal injuries, they nonetheless make active choices about their position within a group. Whilst schooling dynamics have been studied previously in other species this is the first demonstration that physical injury influences an individual's decisions to join groups and to position themselves strategically within them. Our study suggests that for some prey animals, the benefits of grouping outweigh the costs when an injury is recent. However, we observed significant differences in schooling behavior as the injury ages. Over the course of 24 hours, we observed a general decline toward control levels in the propensity for schools to form when an injured squid was present, and in the frequency of school membership for injured squid. However, in the presence of predatory threats, we observed differences in behavior of injured squid to either persist into the long term, or to emerge where they were not present earlier. We did not test the mechanism underlying this change, but it may have been caused by habituation of the group members to the injured animals' presence, habituation of the focal squid's nervous system to the injury (thus removing the proximate neural mechanism that drives behavioral changes), or by changes in risk/benefit profiles of school membership and position, as predation risk declines as injuries seal and cease emitting predator-attracting cues. Future study is needed to establish the underlying mechanisms of the temporal changes we report here.

Whether the presence of an injured member within a group increases the group's overall risk, and thus increases costs for uninjured conspecifics, is not known. We observed no indication that wounded squid were ostracized by uninjured group members, although under some circumstances, injured squid were more likely to occupy peripheral positions within a group. We could not determine if this was a result of the other group members pushing the injured animal to the outside, or an active choice by the injured squid, to either minimize its risk of conspecific predation or maximize its ability to inspect predators. Previous studies of squid schooling in the wild suggest that position and membership decisions depend primarily on the individual, with no observable reaction from school members when joined by a new neighbor (Adamo and Weichelt, 1999). However, given that the presence of an injured conspecific is likely to increase detection and targeting by predators (Crook et al., 2014), it is unclear why school members would accept an injured squid. Although our data show that uninjured squid change their behavior in the presence of an injured conspecific (schooling more frequently), it is unclear whether they can identify the source of the injury cue. Given the small size of the injury, conspecifics may not be able to identify the injured group member directly. Alternatively, uninjured school members may trade off the risk of having an injured group member for the benefits presumably conferred by the injured squid's increased vigilance (Crook et al, 2011, 2014), increasing the net scanning rate of the school. It is also possible that, although having an injured school member increases the frequency of predator interactions with the school, it may decrease individual targeting risk (if the predators focus on the injured squid more frequently and on other group members less frequently).

Asymmetrical predation risks between injured and uninjured group members may explain the positioning decisions made by injured squid. We found that when an olfactory predator cue was presented, the injured squid was more likely to be located on the outside of the group. This implies an active decision by the injured individual to engage in predator inspection behavior (Fitzgibbon, 1994; Godin and Davis, 1995), where a prey animal makes itself conspicuous to, or approaches, a predator, possibly to assess its motivation to engage in pursuit or to deter an ambush predator by indicating it has been detected. 
In contrast, when a predatory threat appeared to be proximate (when squid were exposed to a fish model located with 1 meter of their group), injured squid were more likely to be located in the center of the school. This suggests that once a predatory threat is clearly apparent, there is minimal benefit to the exposure required for further inspection, and instead the injured squid is highly motivated to reduce its risk by choosing a central position where it is shielded by at least one other conspecific.

Our study was conducted in tanks of small size compared to the natural daily range of $D$. pealeii, and the groups of squid we used were also far smaller than the very large school groups that have been observed in this and other Loliginid species. It is therefore possible that the dynamics of larger schools in large spaces may be different. For example, in larger groups there is likely to be more than one squid that is injured, and variations in injury severity and age should be much greater in the field than in our controlled conditions. We show nonetheless that under these conditions, injured squid display significant differences in their schooling behavior compared with uninjured squid.

\section{Conclusions}

Squid with minor fin injury make schooling decisions that differ from those of uninjured squid. Generally, injured squid are more likely to school when predation risk is high. The position of an injured squid within a school depends on the type of potential threat; injured squid prefer peripheral positions when there is no external threat or when an olfactory threat is present, but prefer central positions in the presence of a visual threat. The adaptive choices displayed by injured squid suggest long-lasting cognitive responses to minor injury that have been selected to reduce the enhanced risk of predation that follows sub-lethal injury. 
References:

ABS, 2012. Guidelines for the treatment of animals in behavioural research and teaching. Anim. Behav. 83, 301-309.

Adamo, S.A., Weichelt, K., 1999. Field observations of schooling in the oval squid, Sepioteuthis lessoniana (Lesson, 1830). J. Molluscan Stud. 65, 377-380. doi:10.1093/mollus/65.3.377

Alves, F.L., Barbosa, A., Hoffmann, A., 2013. Antinociception in piauçu fish induced by exposure to the conspecific alarm substance. Physiol. Behav. 110-111, 58-62. doi:10.1016/j.physbeh.2012.12.003

Beauchamp, G., 2015. Animal Vigilance: Monitoring Predators and Competitors. Elsevier Science.

Chivers, D.P., Wisenden, B.D., Hindman, C.J., Michalak, T.A., Kusch, R.C., Kaminskyj, S.G.W., Jack, K.L., Ferrari, M.C.O., Pollock, R.J., Halbgewachs, C.F., Pollock, M.S., Alemadi, S., James, C.T., Savaloja, R.K., Goater, C.P., Corwin, A., Mirza, R.S., Kiesecker, J.M., Brown, G.E., Adrian, J.C., Krone, P.H., Blaustein, A.R., Mathis, A., 2007. Epidermal "alarm substance" cells of fishes maintained by nonalarm functions: possible defence against pathogens, parasites and UVB radiation. Proc. Biol. Sci. 274, 2611-9. doi:10.1098/rspb.2007.0709

Creel, S., Schuette, P., Christianson, D., 2014. Effects of predation risk on group size, vigilance, and foraging behavior in an African ungulate community. Behav. Ecol. 25, 773-784. doi:10.1093/beheco/aru050

Crook, R.J., Dickson, K., Hanlon, R.T., Walters, E.T., 2014. Nociceptive sensitization reduces predation risk. Curr. Biol. 24, 1121-5. doi:10.1016/j.cub.2014.03.043

Crook, R.J., Hanlon, R.T., Walters, E.T., 2013. Squid have nociceptors that display widespread long-term sensitization and spontaneous activity after bodily injury. J. Neurosci. 33, 10021-6. doi:10.1523/JNEUROSCI.0646-13.2013

Crook, R.J., Lewis, T., Hanlon, R.T., Walters, E.T., 2011. Peripheral injury induces longterm sensitization of defensive responses to visual and tactile stimuli in the squid Loligo pealeii, Lesueur 1821. J. Exp. Biol. 214, 3173-85. doi:10.1242/jeb.058131

Fitzgibbon, C.D., 1994. The costs and benefits of predator inspection behaviour in Thomson's gazelles. Behav. Ecol. Sociobiol. 34, 139-148.

Godin, J.-G.J., Davis, S.A., 1995. Who Dares, Benefits: Predator Approach Behaviour in the Guppy (Poecilia reticulata) Deters Predator Pursuit. Proc. R. Soc. B Biol. Sci. 259, 193-200. doi:10.1098/rspb.1995.0028

Hanlon, R.T., Hixon, R.F., Hulet, W.H., 1983. Survival, growth, and behavior of the Ioliginid squids Lologi plei, Loligo pealei, and Lolliguncula brevis (Mollusca: Cephalopoda) in closed sea water systems. Biol. Bull. 637-685.

Herczeg, G., Gonda, A., Merilä, J., 2009. The social cost of shoaling covaries with predation risk in nine-spined stickleback, Pungitius pungitius, populations. Anim. Behav. 77, 575-580. doi:10.1016/j.anbehav.2008.10.023

Hoare, D., Couzin, I., Godin, J., Krause, J., 2004. Context-dependent group size choice in fish. Anim. Behav.

Ibánez, C., Keyl, F., 2010. Cannibalism in cephalopods. Rev. Fish Biol. Fish. 
Kicklighter, C.E., Germann, M., Kamio, M., Derby, C.D., 2007. Molecular identification of alarm cues in the defensive secretions of the sea hare Aplysia californica. Anim. Behav. 74, 1481-1492. doi:10.1016/j.anbehav.2007.02.015

Krause, J., 1993. The effect of "Schreckstoff" on the shoaling behaviour of the minnow: a test of Hamilton's selfish herd theory. Anim. Behav. 45, 1019-1024. doi:10.1006/anbe.1993.1119

Landeau, L., Terborgh, J., 1986. Oddity and the "confusion effect" in predation. Anim. Behav. 34, 1372-1380. doi:10.1016/S0003-3472(86)80208-1

Lutterschmidt, W.I., Marvin, G.A., Hutchison, V.H., 1994. Alarm response by a plethodontid salamander (Desmognathus ochrophaeus): Conspecific and heterospecific "Schreckstoff". J. Chem. Ecol. 20, 2751-9. doi:10.1007/BF02098387

Nikitin, V.P., Kozyrev, S.A., 1999. Neuronal mechanisms of site-specific nociceptive sensitization in the common snail. Neurosci. Behav. Physiol. 29, 167-173. doi:10.1007/BF02465322

Olson, R.S., Hintze, A., Dyer, F.C., Knoester, D.B., Adami, C., 2013. Predator confusion is sufficient to evolve swarming behaviour. J. R. Soc. Interface 10, 20130305. doi:10.1098/rsif.2013.0305

Roberts, G., 1996. Why individual vigilance declines as group size increases. Anim. Behav. 51, 1077-1086. doi:10.1006/anbe.1996.0109

Sauer, W.H.H., Smale, M.J., 1991. Predation patterns on the inshore spawning grounds of the squid Loligo vulgaris reynaudll (Cephalopoda: Loliginidae) off the southeastern Cape, South Africa. South African J. Mar. Sci. 11, 513-523. doi:10.2989/025776191784287736

Sauer, W.H.H., Smale, M.J., Lipinski, M.R., 1992. The location of spawning grounds, spawning and schooling behaviour of the squid Loligo vulgaris reynaudii (Cephalopoda: Myopsida) off the Eastern Cape Coast, South Africa. Mar. Biol. 114, 97-107. doi:10.1007/BF00350859

Staudinger, M.D., Hanlon, R.T., Juanes, F., 2011. Primary and secondary defences of squid to cruising and ambush fish predators: variable tactics and their survival value. Anim. Behav. 81, 585-594.

Staudinger, M.D., Juanes, F., 2010. Size-dependent susceptibility of longfin inshore squid (Loligo pealeii) to attack and capture by two predators. J. Exp. Mar. Bio. Ecol. 393, 106-113. doi:10.1016/j.jembe.2010.07.005

Thünken, T., Eigster, M., Frommen, J.G., 2014. Context-dependent group size preferences in large shoals of three-spined sticklebacks. Anim. Behav. 90, 205210. doi:10.1016/j.anbehav.2014.01.024

Walters, E.T., 1994. Injury-related behavior and neuronal plasticity: an evolutionary perspective on sensitization, hyperalgesia, and analgesia. Int. Rev. Neurobiol. 36, $325-427$.

Walters, E.T., 1987a. Site-specific sensitization of defensive reflexes in Aplysia: a simple model of long-term hyperalgesia. J. Neurosci. 7, 400-407.

Walters, E.T., 1987b. Multiple sensory neuronal correlates of site-specific sensitization in Aplysia. J. Neurosci. 7, 408-417. 
Walters, E.T., Carew, T.J., Kandel, E.R., 1981. Associative learning in Aplysia: evidence for conditioned fear in an invertebrate. Science 211, 504-6.

Walters, E.T., Erickson, M.T., 1986. Directional control and the functional organization of defensive responses in Aplysia. J. Comp. Physiol. A 159, 339-351. doi:10.1007/BF00603980

Wood, J.B., Pennoyer, K.E., Derby, C.D., 2008. Ink is a conspecific alarm cue in the Caribbean reef squid, Sepioteuthis sepioidea. J. Exp. Mar. Bio. Ecol. 367, 11-16. doi:10.1016/j.jembe.2008.08.004 
Figure legends:

Figure 1. Schooling in Doryteuthis pealeii. Top: We defined a schooling group as one that contained at least 3 squid that were oriented in the same direction and positioned within one body length of each other. Additionally, behavioral cohesion (all group members swimming or all resting on the substrate) was a necessary component of any group. Bottom: The focal squid's position within a school was categorized as either central (at least one squid between the focal animal and the school edge), or peripheral (no group members on one side of the focal animal). When the focal animal was on the edge of a group, we further classified its position as either "treated side in" or "treated side out."

Figure 2. A: Proportion of time a school was present after one member sustained a minor injury, irrespective of whether the treated squid was a member of the school. A. Short-term (30min-2h) time points, B long-term (6-24h) time points. Bottom: Proportion of time the focal squid was a member of a schooling group. C. Short term, D. Long term. Bars show mean +1 SEM. ${ }^{*} p<0.05,{ }^{* *} p<0.01,{ }^{* * *} p<0.005$ (Mann-Whitney-U tests).

Figure 3. Top: Proportion of time the focal squid was on the edge of the school. A. Short-term (30min-2h) time points, B long-term (6-24h) time points. Bottom:

Proportion of time the treated side faced the outside of the school. C. Short term, D. Long term. Bars show mean +1 SEM. * $p<0.05$ (Mann-Whitney-U tests). 

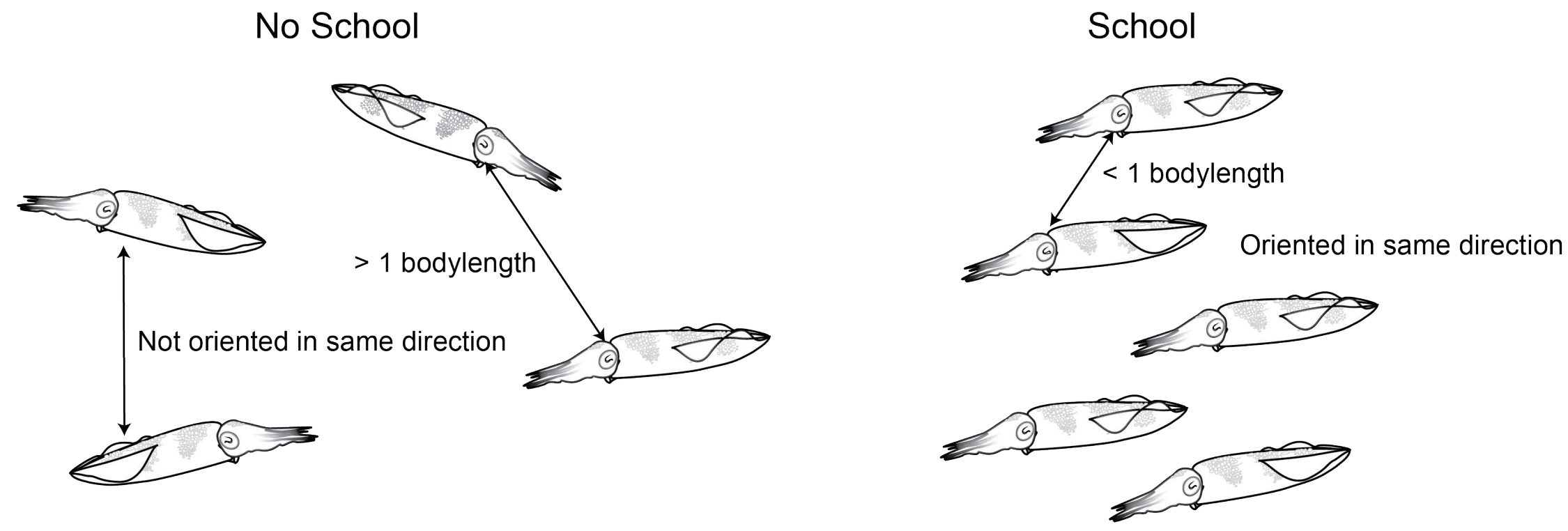

Focal squid in center
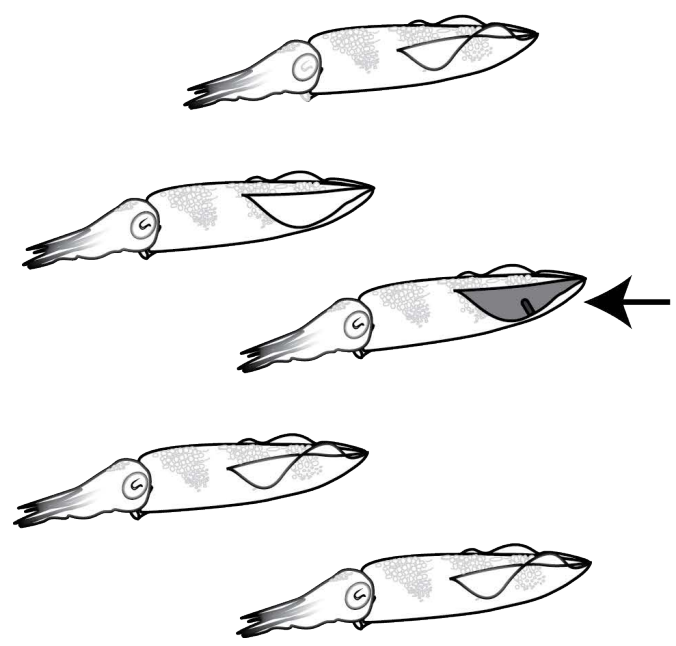

Focal squid on edge, treated side out
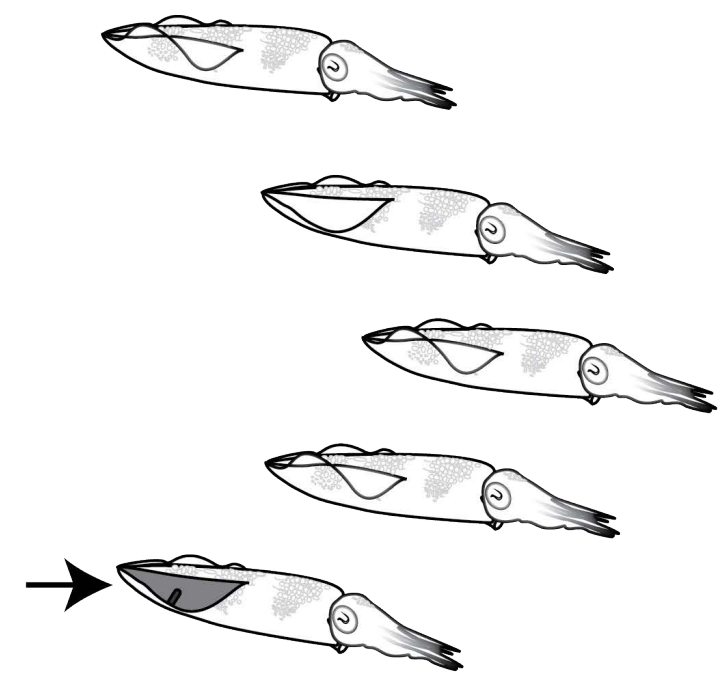

Focal squid on edge, treated side in

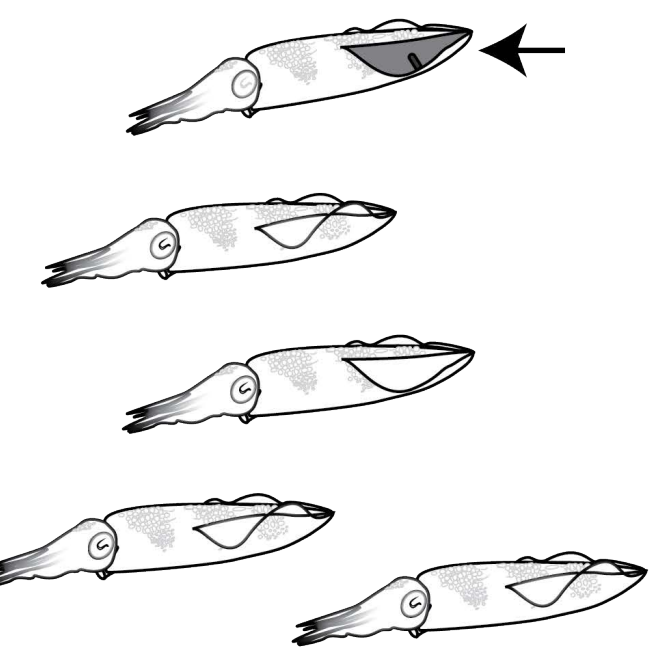


Short term

Long term

Proportion of time there is a school

A

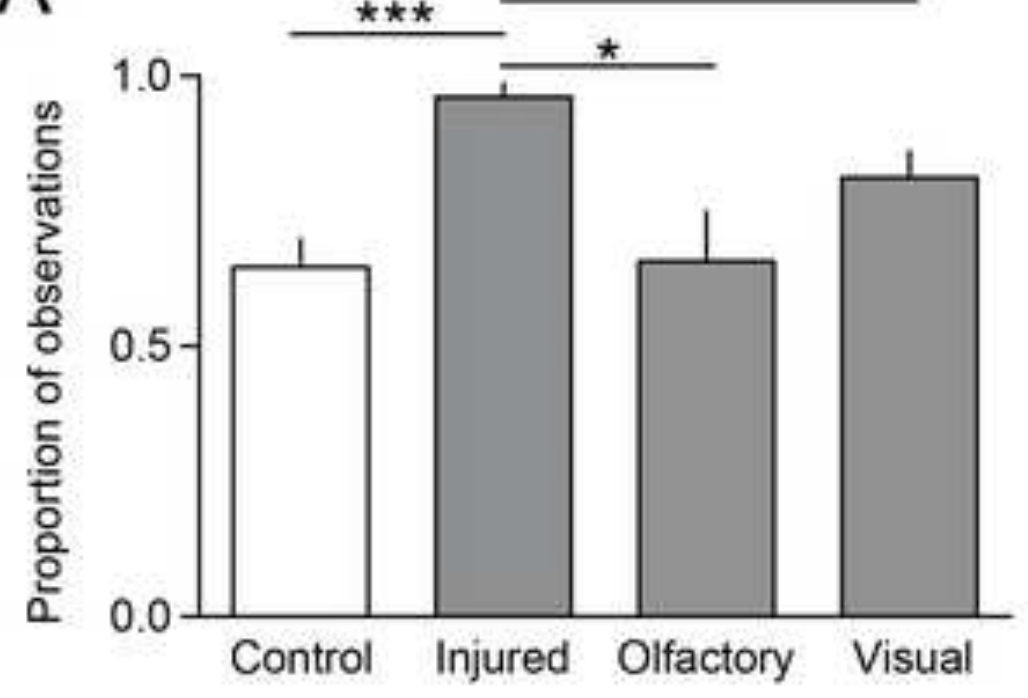

B

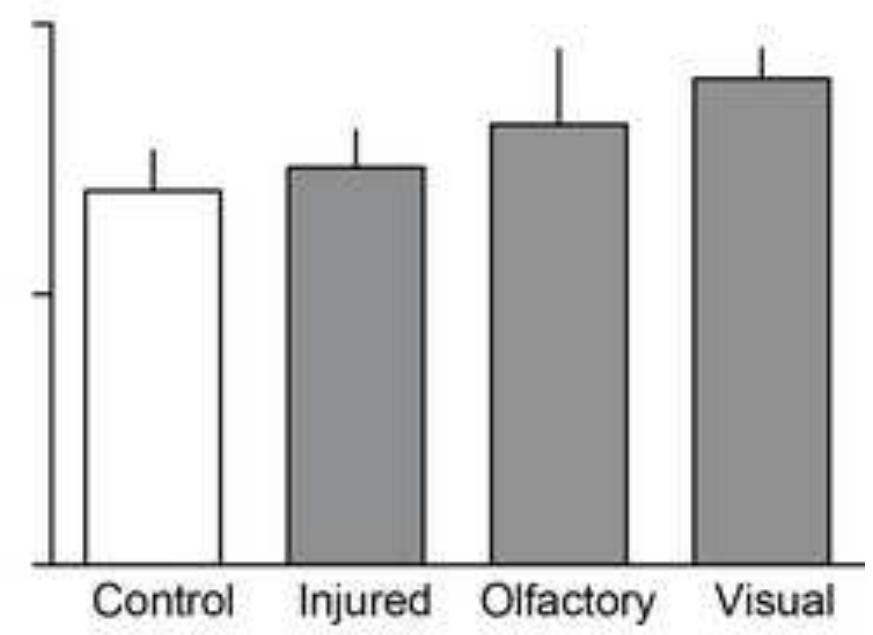

Proportion of time squid is in the school

C

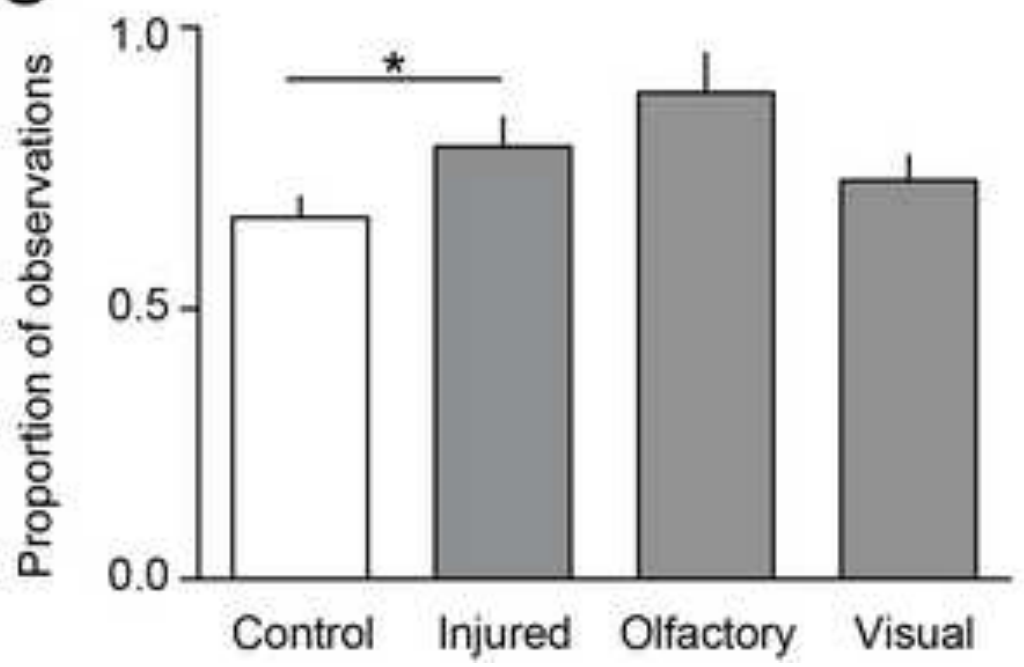

D

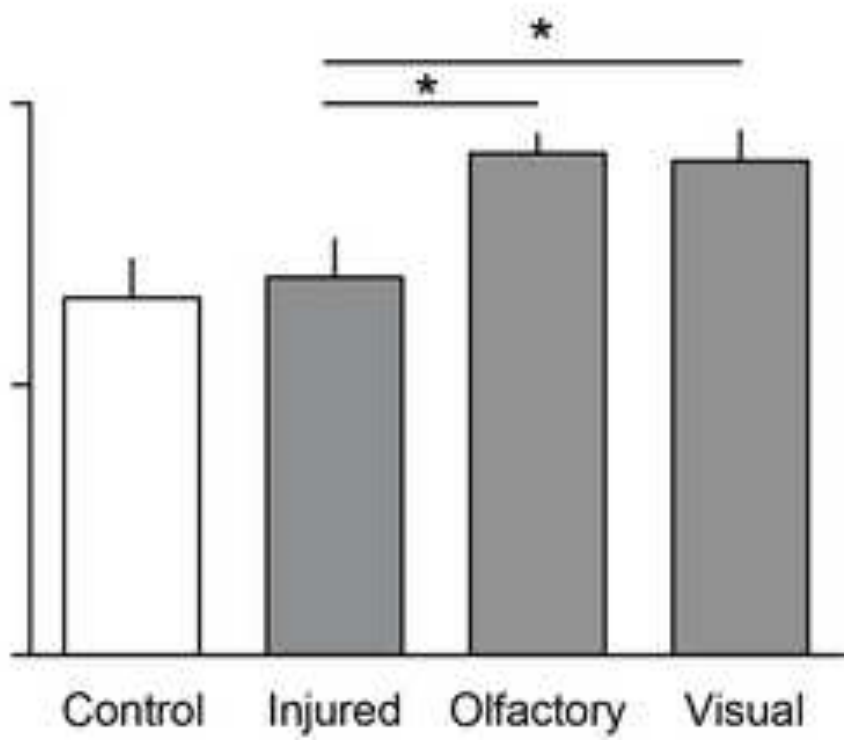




\section{Short term}

\section{Long term}

Proportion of time squid is on the edge of school

A

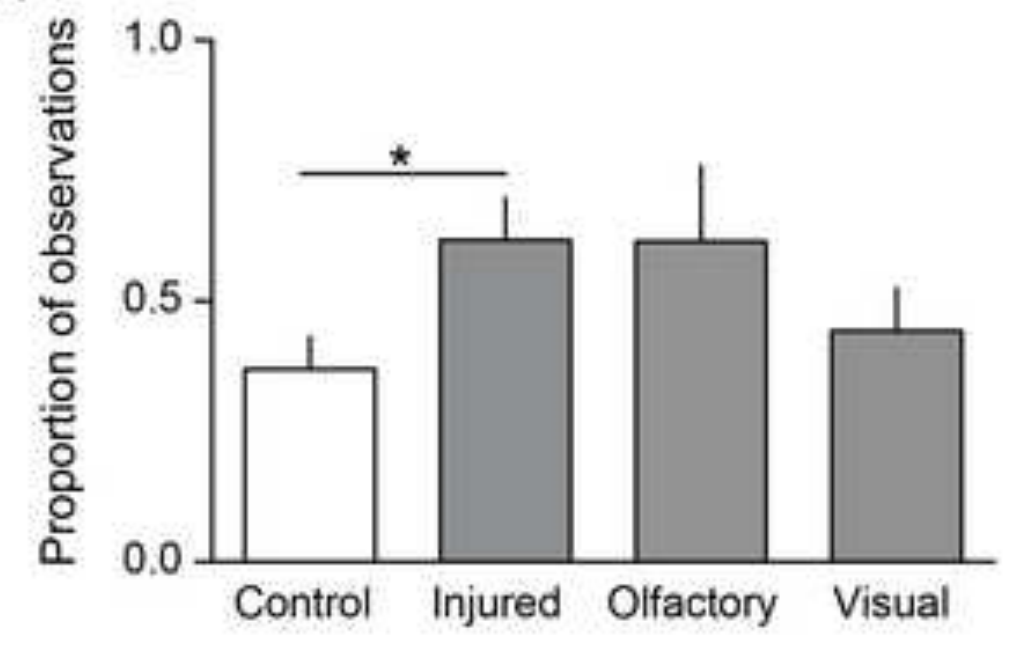

B

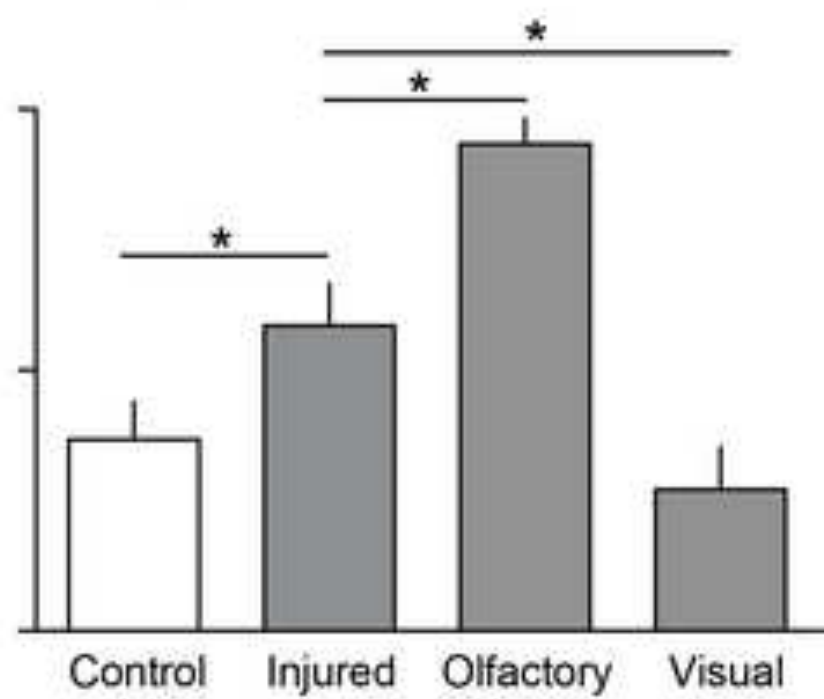

\section{Proportion of time treated side faces out}

C

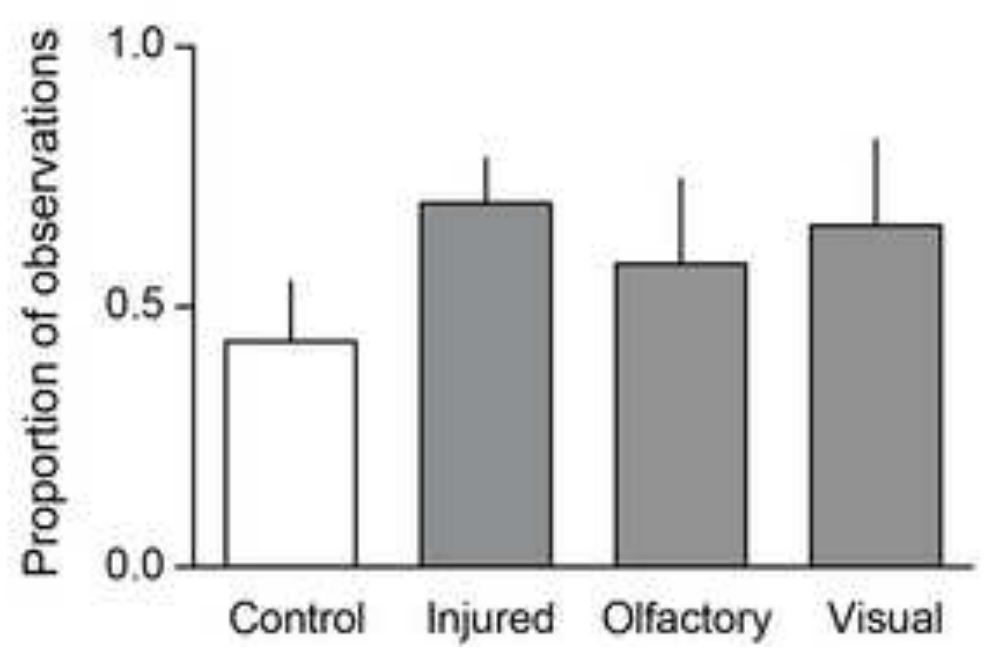

D

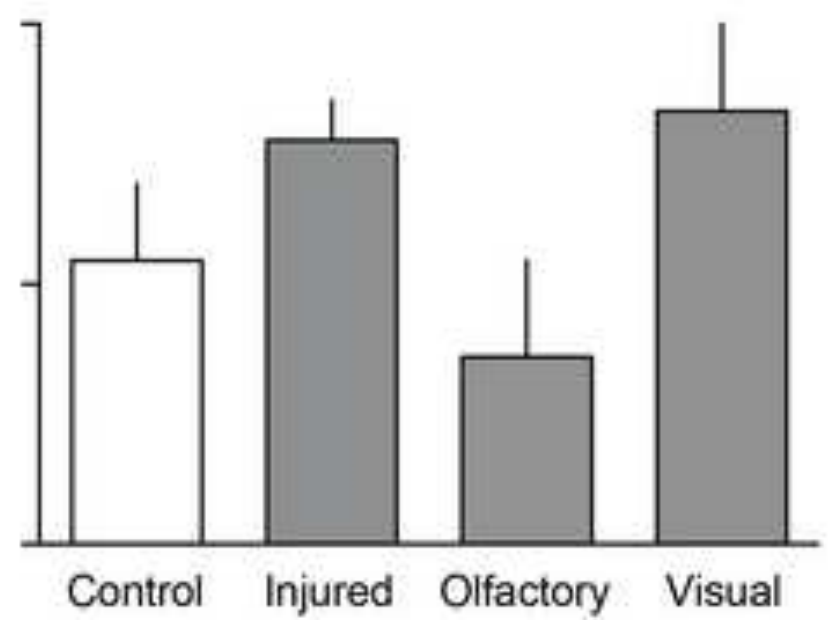

